\title{
Nuclear Astrophysics in underground laboratories: the LUNA experiment
}

\author{
Antonio Caciolli for the LUNA Collaboration ${ }^{1, \star}$ \\ ${ }^{1}$ University and INFN of Padua, via Marzolo 8, 35131, Padua, Italy
}

\begin{abstract}
One of the main ingredients of nuclear astrophysics is the knowledge of the thermonuclear reactions responsible for powering the stellar engine and for the synthesis of the chemical elements. At astrophysical energies the cross section of nuclear processes is extremely reduced by the effect of the Coulomb barrier. The low value of cross sections prevents their measurement at stellar energies on Earth surface and often extrapolations are needed. The Laboratory for Underground Nuclear Astrophysics (LUNA) is placed under the Gran Sasso mountain and thanks to the cosmic-ray background reduction provided by its position can investigate cross sections at energies close to the Gamow peak in stellar scenarios. Many crucial reactions involved in hydrogen burning has been measured directly at astrophysical energies with both the LUNA-50kV and the LUNA-400kV accelerators, and this intense work will continue with the installation of a MV machine able to explore helium and carbon burnings. Based on this progress, currently there are efforts in several countries to construct new underground accelerators. In this talk, the typical techniques adopted in underground nuclear astrophysics will be described and the most relevant results achieved by LUNA will be reviewed. The exciting science that can be probed with the new facilities will be highlighted.
\end{abstract}

Nuclear processes generate the energy that makes stars shine. Moreover they are responsible of the synthesis of the elements (and isotopes) in stars. As a matter of fact, hydrogen, helium and all isotopes until lithium and beryllium are synthesised during the Big Bang Nucleosynthesis $(\mathrm{BBN})$. All other nuclei are produced during the different characteristic phases of the star evolution [1].

The understanding of these nuclear processes is the goal of nuclear astrophysics and, in particular, the knowledge of the nuclear cross-sections involved in that processes. The astrophysical relevant energies is given by the folding of two strongly energy dependent functions [1, 2]: the Maxwell-Boltzmann velocity distribution and the cross section of charged particle induced reactions. The folding results in a structure called the Gamow peak, which peaks at the energy $E_{G}$ (called Gamow energy):

$$
E_{G} \approx 0.1220\left(Z_{1}^{2} Z_{2}^{2} \mu\right)^{\frac{1}{3}} T_{9}^{\frac{2}{3}}
$$

where $Z_{1,2}$ are the charges of the two reaction partners, $\mu=$ $m_{1} m_{2} /\left(m_{1}+m_{2}\right)$ their reduced mass, and $T_{9}=T / 10^{9} \mathrm{~K}$ is the temperature of the astrophysical scenario under study. The Gamow energy of nuclear reactions taking place in the Sun, with its core temperature of $T_{9} \approx 0.016$, is typically $20 \mathrm{keV}$ depending on the precise reaction, leading to cross sections in the range of pbarn and below. As matter of fact, in this energy range the cross section is highly reduced by the effect of the Coulomb repulsion and the nuclear reactions can occur only via tunnel effect. In particular the

\footnotetext{
^e-mail: caciolli@pd.infn.it
}

cross section can be written as:

$$
\sigma(E)=\frac{S(E)}{E} \exp (-2 \pi \eta)
$$

where $S(E)$ is the astrophysical factor (which contains the nuclear physics information) and $\eta$ is the Sommerfeld parameter, given by:

$$
2 \pi \eta=31.29 Z_{1} Z_{2}(\mu / E)^{1 / 2},
$$

$\mu$ is the reduced mass (in units of amu), and $E$ is the center of mass energy (in units of keV).

Due to these small cross section values, the rate of the reactions, characterised by a typical energy release of a few $\mathrm{MeV}$, is too low, down to a few events per year, in order to stand out from the laboratory background. In many cases it is not possible even to reach energy values close to the Gamow peak and extrapolations are needed, leading to substantial uncertainties. A way to handle that background problem is to go in an underground environment [3]. As a matter of fact the natural shielding provided by an underground site will guarantee a reduction of the cosmic flux of orders of magnitude leading to the success of experimental nuclear physics. LUNA (Laboratory for Underground Nuclear Physics) [4, 5] is placed under the Gran Sasso National Laboratories of INFN. Two accelerators were used during years. First a $50 \mathrm{kV}$ accelerator (hereafter LUNA1)[6] and then a $400 \mathrm{kV}$ accelerator (hereafter LUNA2)[7].

Under the Gran Sasso National Laboratory (LNGS) the muon flux is reduced by a factor $10^{6}$ and the neutron flux by a factor of 1000 [8, 9]. Further background re- 
duction in the region below $3 \mathrm{MeV}$ in the gamma spectrum can be achieved by implementing a shielding made by copper and lead $[10,11]$. A comparison between two gamma spectra acquired with the same detector in surface and at LNGS is shown in Figure 1. In the figure, the detector was also shielded by few $\mathrm{cm}$ of OFHC copper and $25 \mathrm{~cm}$ of low level background lead when placed in the underground laboratory to obtain almost 4 order of magnitude reduction in the $\gamma$-ray background below $3 \mathrm{MeV}$ [10]. A review of the results achieved by the LUNA col-

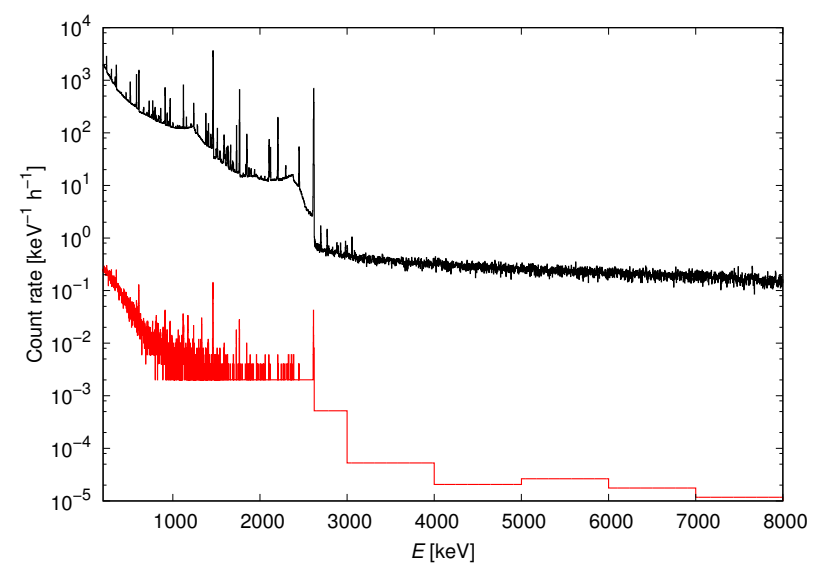

Figure 1. The spectrum acquired with an HPGe on the surface in Padua (black) is compared with the one acquried with same detector in the underground laboratory of Gran Sasso and with a full shield as reported in [10] (red).

laboration will be presented in this paper combined with a discussion on the future projects for nuclear astrophysics in underground with a MV accelerator.

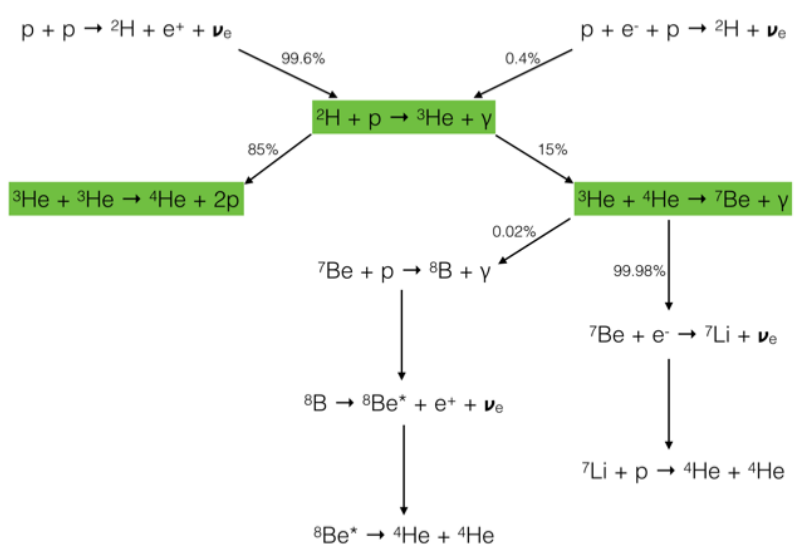

Figure 2. Reactions scheme of the p-p chain. The reaction studied by LUNA are highlighted in green.

\section{Solar Hydrogen Burning}

Hydrogen burning in the Sun proceeds mainly by the proton-proton chain (pp-chain, Fig. 2), with a $0.8 \%$ contribution from the carbon-nitrogen-oxygen cycle ( $\mathrm{CN}$ cycle) [12]. The basic processes are by now well understood, leading to the so-called standard solar model [13] that ex- plains both helioseismological data and neutrino observations.

LUNA started its work studying the ${ }^{3} \mathrm{He}\left({ }^{3} \mathrm{He}, 2 \mathrm{p}\right){ }^{4} \mathrm{He}$ reaction. This is a key reaction of the hydrgen burning proton proton chain and at the beginning of the seventies it was believed to exist a possible resonance in the ${ }^{3} \mathrm{He}\left({ }^{3} \mathrm{He}, 2 \mathrm{p}\right){ }^{4} \mathrm{He}$ Gamow peak that could have explained the low measured neutrino flux measured by the Homestake experiment [14]. The experiment was performed at the LUNA1 accelerator by using a windowless gas target, filled with ${ }^{3} \mathrm{He}$, and eight $1 \mathrm{~mm}$ thick silicon detectors of $5 \times 5 \mathrm{~cm}^{2}$ area. The cross section was measured in an energy range from 16.5 to $24.4 \mathrm{keV}$, totally covering the Gamow peak for the Sun $[15,16]$. The debated resonance $[17,18]$ was not observed, ruling out the possible nuclear explanation of the so called Solar Neutrino Problem.

The neutrino fluxes emitted by the Sun are strictly correlated with other nuclear processes involved in the hydrogen burning. In particular, the ${ }^{3} \mathrm{He}(\alpha, \gamma){ }^{7} \mathrm{Be}$ reaction reaction controls the ${ }^{7} \mathrm{Be}$ and ${ }^{8} \mathrm{~B}$ neutrinos and it is also fundamental for the production of ${ }^{7} \mathrm{Li}$ during the Big Bang Nucleosynthesis (BBN). The ${ }^{3} \mathrm{He}(\alpha, \gamma)^{7} \mathrm{Be}$ reaction was studied at the LUNA2 accelerator by using both the gamma prompt detection $[19,20]$ and the activation techniques $[21,22]$ finding a perfect agreement within the two methods. This result was important not only to reduce the systematics, but also to solve the discrepancy previously shown in experiments based on the two different techniques.

The ${ }^{14} \mathrm{~N}(\mathrm{p}, \gamma){ }^{15} \mathrm{O}$ reaction is the bottleneck of the $\mathrm{CN}$ cycle and therefore the ${ }^{13} \mathrm{~N}$ and ${ }^{15} \mathrm{O}$ neutrinos are controlled by this reaction. The LUNA collaboration started the study of this reaction by performing two different experiments: one characterised by solid target setup and HPGe detector. This kind of setup allows to study separately all branchings and to perform R-Matrix analysis $[23,24]$. A second setup, made of a windowless gas target and a $4 \pi$-BGO detector, was used to measure the total cross section in a wide range of energy (down to $70 \mathrm{keV}$ in the center of mass) thanks to the high efficiency provided by the BGO $[25,26]$, totally covering the nova explosion scenario with experimental data. In those experiments the cross section was found lower by a factor of 2 with respect to the reported value in NACRE [27] with considerably effects on the neutrino fluxes from the Sun and the age of globular clusters[28].

Recently, a discrepancy between helioseismology and the new metal abundances (i.e. the amount of elements different from hydrogen and helium) that emerged from improved modelling of the photosphere has been reported [29]. This so called Solar Composition Problem [12] can be addressed by the possibility to detect the CNO neutrino with new experiment and to parametrise with extreme precision the Solar models. For this reason the ${ }^{14} \mathrm{~N}(\mathrm{p}, \gamma){ }^{15} \mathrm{O}$ cross section is requested to be known with a precision of $5 \%$, almost factor of two lower than what reported in the last database [30]. In order to reduce this nuclear uncertainties in the solar model a new measurement was performed at LUNA by measuring three energy point above the $259 \mathrm{keV}$ resonance and leading to and extrapolated 
value $\mathrm{S}_{1,14}(0)=1.57 \pm 0.13 \mathrm{keV}$ barn $[31,32]$. Another recent measurements has been performed at the Notre Dame University [33] at higher energies obtaining an extrapolated value higher than the one reported by LUNA. In this context a new measurement is requested in a wide energy range and it will be done as first measurement of the future LUNA MV accelerator, which will cover an energy range from $3 \mathrm{MeV}$ down to $200 \mathrm{keV}$ with an unique set of data, leading to precise extrapolation.

\subsection{The other CNO cycles}

The ${ }^{15} \mathrm{~N}(\mathrm{p}, \gamma){ }^{16} \mathrm{O}$ is the link from the first to the second CNO cycle and it was studied intensively at the LUNA accelerator. Data acquired with a gas target setup and natural nitrogen (which contains a $0.37 \%$ of ${ }^{15} \mathrm{~N}$ ) to study the ${ }^{14} \mathrm{~N}(\mathrm{p}, \gamma){ }^{15} \mathrm{O}$ reaction were analysed to determine the ${ }^{14} \mathrm{~N}(\mathrm{p}, \gamma){ }^{15} \mathrm{O}$ cross section in the energy range from 230 $\mathrm{keV}$ down to $90 \mathrm{keV}$ in the center of mass[34]. After that, two new experiments were performed by using ${ }^{15} \mathrm{~N}$ enriched solid targets $[35,36]$. The LUNA data cover totally the Gamow peak in nova explosion where the ${ }^{15} \mathrm{~N}(\mathrm{p}, \gamma){ }^{16} \mathrm{O}$ is mainly important and the cross section was found to be lower than a factor of 2 with respect what reported in the NACRE database [27]. This leads to a reduction of ${ }^{16} \mathrm{O}$ produced by nova of a $40 \%$.

The ${ }^{17} \mathrm{O}(\mathrm{p}, \gamma){ }^{18} \mathrm{~F}$ reaction was investigated from 2011 to 2013. The ratio between the reaction rates of ${ }^{17} \mathrm{O}(\mathrm{p}, \alpha){ }^{14} \mathrm{~N}(\mathrm{Q}=1.2 \mathrm{MeV})$ and ${ }^{17} \mathrm{O}(\mathrm{p}, \gamma){ }^{18} \mathrm{~F}(\mathrm{Q}=5.6$ $\mathrm{MeV}$ ) channels is one of the most important parameters for the galactic synthesis of ${ }^{17} \mathrm{O}$, the stellar production of radioactive ${ }^{18} \mathrm{~F}$, and for predicted $\mathrm{O}$ isotopic ratios in premolar grains $[37,38]$.

Since the ${ }^{18} \mathrm{~F}$ is a radionuclides with a half life of $\approx 110 \mathrm{~min}$, the cross section has been determined both by detecting the prompt gamma rays and by counting the $511 \mathrm{keV} \gamma \mathrm{s}$ emitted by the ${ }^{18} \mathrm{~F}$ decay. The results are in perfect agreement reducing considerably the systematics [39]. New results on the non resonant component and on the $183.3 \mathrm{keV}$ resonance have been obtained $(\omega \gamma=(1.67 \pm 0.12) \mu \mathrm{eV}[39,40])$ reducing the uncertainties of isotopes production in nova explosion models to a $10 \%$ level [40]. The very low uncertainty obtained in this experiment was possible thank to an intensive study of the target, realised and tested directly by the LUNA group with ion beam analysis and secondary induced mass spectroscopy techniques [41].

An intense study on the ( $\mathrm{p}, \alpha)$ channel has been followed: a detection system composed by eight silicon detectors was used in this study to detect with high efficiency the $\alpha$ particles emitted. This experimental system is described in [42] and was used to measure directly the low energy resonance at $64.5 \mathrm{keV}$ finding an $\omega \gamma=\left(8.7 \pm 1.2_{\text {stat }} \pm 0.6_{\text {syst }}\right) \mathrm{neV}$ for the bare nuclei [43]. The LUNA value is a factor of 2 higher than what previously reported in literature $[44,45]$ bringing to important consequences in the AGB [46] and, in particular, in finding a possible explanation for the production sites of pre-solar grains enriched in ${ }^{17} \mathrm{O}$ [47].

\subsection{The $\mathrm{Ne}-\mathrm{Na}$ and $\mathrm{Mg}$-Al cycles at LUNA}

The nucleosynthesis in the neon sodium cycle occurs via many reactions. In particular, the uncertainty on the ${ }^{22} \mathrm{Ne}(\mathrm{p}, \gamma)^{23} \mathrm{Na}$ dominates the uncertainty of the entire cycle. As a matter of fact this reaction has several resonances below $400 \mathrm{keV}$ and many of them were only given as upper limits based on indirect measurements. In particular, the databases [27, 48] reported a discrepancy of a factor one thousand. A fully shielded setup has been implemented [11] to observe for the first time three resonances and to improve the upper limits for other three resonances $[49,50]$. Finally, an updated reaction rate has been obtained, which is significantly higher than the one given in the most recent compilation of reaction rates [48]. As a consequence, new values for the ejected mass of ${ }^{22} \mathrm{Ne}$ and ${ }^{23} \mathrm{Na}$ in thermally pulsing AGB stars have been obtained with much reduced uncertainties [51].

The study of the CNO cycles is the natural precursor for the hydrogen burning in $\mathrm{Mg}$ - $\mathrm{Al}$ cycles. The problem of the ${ }^{26} \mathrm{Al}$ production is one of the most interesting cases [52]. LUNA measured precisely several resonances for ${ }^{24,25} \mathrm{Mg}(\mathrm{p}, \gamma)^{26,27} \mathrm{Al}$ in order to reduce the uncertainties on those reactions as requested in the astrophysical models $[53,54]$. The impact of the LUNA results is discussed in details in a recent work [55].

\section{Big Bang Nucleosynthesis}

The ${ }^{3} \mathrm{He}(\alpha, \gamma)^{7} \mathrm{Be}$ reaction has an important role in solving the problem of the Spite plateau [56]. LUNA measured this reaction in the Gamow peak for Big Bang Nucleosynthesis reducing the uncertainties to $3 \%$ overall. Another problem concerning lithium isotopes as been raised recently: the ${ }^{6} \mathrm{Li}$ has been measured to be 3 order of magnitude higher than what expected in BBN [57]. While it is generally believed that ${ }^{6} \mathrm{Li}$ is produced by cosmic-ray spallation over the lifespan of the universe, the ${ }^{6} \mathrm{Li}$ observations in halo stars seem to suggest a primordial origin instead. For ${ }^{6} \mathrm{Li}$ production in the Big Bang, the main nuclear physics unknown is the ${ }^{2} \mathrm{H}(\alpha, \gamma)^{6} \mathrm{Li}$ reaction rate. The setup used to study this reaction is already described in details [58]. The scattering chamber was filled with high purity deuterium gas. The accelerated deuterium particles, after Rutherford scattering with the $\alpha$-beam, interact with the gas itself producing the two reactions: ${ }^{2} \mathrm{H}(\mathrm{d}, \mathrm{n})^{3} \mathrm{He}$ and ${ }^{2} \mathrm{H}(\mathrm{d}, \mathrm{p})^{3} \mathrm{H}$. In particular, the neutrons emitted by the ${ }^{2} \mathrm{H}(\mathrm{d}, \mathrm{n}){ }^{3} \mathrm{He}$ were responsible for a high beam induced background in the $\gamma$-spectrum. A long and detailed study of this background was required in order to perform the analysis of the data [58-60].

\section{Science case for a future higher-energy accelerator underground}

Recent advances in astronomy and astrophysics require nuclear data at energies that are higher than the highenergy limit of LUNA2.

To explore the helium and carbon burnings a new MV accelerator will be installed at LNGS during the 2018 . 
With the new accelerator, a long list of reactions will be studied. A first 5 years program is detailed discussed in [61]. The first reaction will be the ${ }^{14} \mathrm{~N}(\mathrm{p}, \gamma){ }^{15} \mathrm{O}$. The solar composition problem has been already addressed above and recently a new measurement has been performed at high energy founding discrepancies with the LUNA data [33]. With the new LUNA MV it will be possibile to cover with an unique set of data a wide energy range from 2 $\mathrm{MeV}$ down to the LUNA2 energies, allowing to reduce the systematics down to the goal of $5 \%$.

Another important open issue of nuclear astrophysics is the neutron source reactions. In particular the ${ }^{13} \mathrm{C}(\alpha, \mathrm{n}){ }^{16} \mathrm{O}$ and ${ }^{22} \mathrm{Ne}(\alpha, \mathrm{n}){ }^{25} \mathrm{Mg}$ reactions. They are responsible for the production on neutrons involved in the slow neutron capture process, called the astrophysical sprocess. Whereas those reactions are the subject of intensive experimental study [62, e.g.], so far the reactions actually producing the neutrons have not yet been measured in the relevant energy range since they should be addressed by an underground accelerator.

The ${ }^{12} \mathrm{C}+{ }^{12} \mathrm{C}$ is the trigger of the carbon burning and is a key reaction to predict the final stage of a star. It determines which is the mass required to a star to ignite this burning stage. In addition, this reaction also affects the outcome of type Ia supernovae. Both the two channels (proton and $\alpha$ ) have never been studied down below $2 \mathrm{MeV}$. At the LUNA MV it will be possible to measure the cross section down to $1.7 \mathrm{MeV}$ very close to cover the whole Gamow peak.

A third topic is to complement some of the protonand $\alpha$-capture reactions studied at the LUNA2 accelerator at higher energy. Such a continuation is particularly important for the Big Bang reactions ${ }^{3} \mathrm{He}(\alpha, \gamma)^{7} \mathrm{Be}$ and ${ }^{2} \mathrm{H}(\alpha, \gamma)^{6} \mathrm{Li}$, where the present LUNA2 $400 \mathrm{kV}$ machine can only cover the lower part of the relevant energy region.

\section{Future underground accelerator facilities}

Based on the successes of the LUNA collaboration, around the world several efforts are underway to install highcurrent, stable-beam accelerators in underground sites. LUNA MV project was started in order to install a $3.5 \mathrm{MV}$ machine in the underground laboratories of Gran Sasso. The new accelerator has already been financed by the Italian government and it should be installed in the next year at the Gran Sasso Laboratory. The synergy between the existing LUNA2 and the new LUNA MV accelerator will allow to perform reaction studies in a wide range of energies with complete understanding of the setups involved. Other facilities have been proposed for installing an accelerator in deep underground laboratory in US [63] and in China [64]. Another project is under discussion at the Canfranc underground laboratory in the Pyrenees, Spain. As part of a staged approach, even an accelerator laboratory in a shallow-underground facility such as Felsenkeller (Dresden, Germany) is under consideration.

At present, the existing $400 \mathrm{kV}$ LUNA2 machine continues its scientific program outlined in [65]. The next few years will show where this highly successful approach will eventually be complemented by one or more higherenergy accelerators underground. The technique is sufficiently mature to address not only the data needs of the astrophysics community, but it has the potential to benefit also the astroparticle and other communities.

\section{Acknowledgments}

The work at LUNA is carried out in the framework of the LUNA collaboration; see e.g. Ref. [43] for an author list.

\section{References}

[1] C. Iliadis, Nuclear Physics of Stars (Wiley-VCH, Weinheim, 2007)

[2] C. Rolfs, W. Rodney, Cauldrons in the Cosmos (University of Chicago Press, Chicago, 1988)

[3] Best, A., Caciolli, A., F|"ulüp, Zs., Gy|"orky, Gy., Laubenstein, M., Napolitani, E., Rigato, V., Roca, V., Sz|"ucs, T., Eur. Phys. J. A 52, 72 (2016)

[4] H. Costantini, A. Formicola, G. Imbriani, M. Junker, C. Rolfs, F. Strieder, Reports on Progress in Physics 72, 086301 (2009)

[5] C. Broggini, D. Bemmerer, A. Guglielmetti, R. Menegazzo, Annu. Rev. Nucl. Part. Sci. 60, 53 (2010)

[6] U. Greife, C. Arpesella, C. Barnes, F. Bartolucci, E. Bellotti, C. Broggini, P. Corvisiero, G. Fiorentini, A. Fubini, G. Gervino et al., Nucl. Inst. Meth. A 350, 327 (1994)

[7] A. Formicola, G. Imbriani, M. Junker, D. Bemmerer, R. Bonetti, C. Broggini, C. Casella, P. Corvisiero, H. Costantini, G. Gervino et al., Nucl. Inst. Meth. A 507, 609 (2003)

[8] D. Bemmerer, F. Confortola, A. Lemut, R. Bonetti, C. Broggini, P. Corvisiero, H. Costantini, J. Cruz, A. Formicola, Z. Fülöp et al., Eur. Phys. J. A 24, 313 (2005)

[9] T. Szücs, D. Bemmerer, C. Broggini, A. Caciolli, F. Confortola, P. Corvisiero, Z. Elekes, A. Formicola, Z. Fülöp, G. Gervino et al., Eur. Phys. J. A 44, 513 (2010)

[10] A. Caciolli, L. Agostino, D. Bemmerer, R. Bonetti, C. Broggini, F. Confortola, P. Corvisiero, H. Costantini, Z. Elekes, A. Formicola et al., Eur. Phys. J. A 39, 179 (2009)

[11] F. Cavanna, R. Depalo, M.L. Menzel, M. Aliotta, M. Anders, D. Bemmerer, C. Broggini, C.G. Bruno, A. Caciolli, P. Corvisiero et al., Eur. Phys. J. A 50, 179 (2014), 1411.2888

[12] A.M. Serenelli, S. Basu, J.W. Ferguson, M. Asplund, Astrophys. J. Lett. 705, L123 (2009), 0909. 2668

[13] J.N. Bahcall, M.H. Pinsonneault, Phys. Rev. Lett. 92 , 121301 (2004)

[14] R. Davis, D.S. Harmer, K.C. Hoffman, Phys. Rev. Lett. 20, 1205 (1968) 
[15] M. Junker, A. D'alessandro, S. Zavatarelli, C. Arpesella, E. Bellotti, C. Broggini, P. Corvisiero, G. Fiorentini, A. Fubini, G. Gervino et al., Phys. Rev. C 57, 2700 (1998), nucl-ex/9707003

[16] R. Bonetti, C. Broggini, L. Campajola, P. Corvisiero, A. D'Alessandro, M. Dessalvi, A. D'Onofrio, A. Fubini, G. Gervino, L. Gialanella et al., Phys. Rev. Lett. 82, 5205 (1999), arXiv: nucl-ex/9902004

[17] W.A. Fowler, Nature 238, 24 (1972)

[18] V.N. Fetisov, Y.S. Kopysov, Phys. Lett. B 40, 602 (1972)

[19] D. Bemmerer, F. Confortola, H. Costantini, A. Formicola, G. Gyürky, R. Bonetti, C. Broggini, P. Corvisiero, Z. Elekes, Z. Fülöp et al. (LUNA Collaboration), Phys. Rev. Lett. 97, 122502 (2006)

[20] G. Gyürky, F. Confortola, H. Costantini, A. Formicola, D. Bemmerer, R. Bonetti, C. Broggini, P. Corvisiero, Z. Elekes, Z. Fülöp et al. (LUNA Collaboration), Phys. Rev. C 75, 035805 (2007)

[21] F. Confortola, D. Bemmerer, H. Costantini, A. Formicola, G. Gyürky, P. Bezzon, R. Bonetti, C. Broggini, P. Corvisiero, Z. Elekes et al. (LUNA Collaboration), Phys. Rev. C 75, 065803 (2007)

[22] H. Costantini, D. Bemmerer, F. Confortola, A. Formicola, G. Gyürky, P. Bezzon, R. Bonetti, C. Broggini, P. Corvisiero, Z. Elekes et al., Nucl. Phys. A 814, 144 (2008), 0809. 5269

[23] A. Formicola, G. Imbriani, H. Costantini, C. Angulo, D. Bemmerer, R. Bonetti, C. Broggini, P. Corvisiero, J. Cruz, P. Descouvemont et al., Phys. Lett. B 591, 61 (2004)

[24] G. Imbriani, H. Costantini, A. Formicola, A. Vomiero, C. Angulo, D. Bemmerer, R. Bonetti, C. Broggini, F. Confortola, P. Corvisiero et al., Eur. Phys. J. A 25, 455 (2005)

[25] A. Lemut, D. Bemmerer, F. Confortola, R. Bonetti, C. Broggini, P. Corvisiero, H. Costantini, J. Cruz, A. Formicola, Z. Fülöp et al., Phys. Lett. B 634, 483 (2006), nucl-ex/0602012

[26] D. Bemmerer, F. Confortola, A. Lemut, R. Bonetti, C. Broggini, P. Corvisiero, H. Costantini, J. Cruz, A. Formicola, Z. Fülöp et al., Nucl. Phys. A 779, 297 (2006)

[27] C. Angulo, M. Arnould, M. Rayet, P. Descouvemont, D. Baye, C. Leclercq-Willain, A. Coc, S. Barhoumi, P. Aguer, C. Rolfs et al., Nucl. Phys. A 656, 3 (1999)

[28] Imbriani, G., Costantini, H., Formicola, A., Bemmerer, D., Bonetti, R., Broggini, C., iero, P. Corvi, Cruz, J., Fülop, Z., Gervino, G. et al., A\&A 420, 625 (2004)

[29] C. Peña-Garay, A. Serenelli, ArXiv e-prints (2008), 0811.2424

[30] E. Adelberger, A. García, R.G.H. Robertson, K.A. Snover, A.B. Balantekin, K. Heeger, M.J. RamseyMusolf, D. Bemmerer, A. Junghans, C.A. Bertulani et al., Rev. Mod. Phys. 83, 195 (2011)

[31] M. Marta, A. Formicola, G. Gyürky, D. Bemmerer, C. Broggini, A. Caciolli, P. Corvisiero, H. Costan- tini, Z. Elekes, Z. Fülöp et al., Phys. Rev. C 78, 022802(R) (2008)

[32] M. Marta, A. Formicola, D. Bemmerer, C. Broggini, A. Caciolli, P. Corvisiero, H. Costantini, Z. Elekes, Z. Fülöp, G. Gervino et al., Phys. Rev. C 83, 045804 (2011), 1103.5393

[33] Q. Li, J. Görres, R.J. deBoer, G. Imbriani, A. Best, A. Kontos, P.J. LeBlanc, E. Uberseder, M. Wiescher, Phys. Rev. C 93, 055806 (2016)

[34] D. Bemmerer, A. Caciolli, R. Bonetti, C. Broggini, F. Confortola, P. Corvisiero, H. Costantini, Z. Elekes, A. Formicola, Z. Fülöp et al., J. Phys. G 36, 045202 (2009), 0902.0783

[35] P.J. LeBlanc, G. Imbriani, J. Görres, M. Junker, R. Azuma, M. Beard, D. Bemmerer, A. Best, C. Broggini, A. Caciolli et al., Phys. Rev. C 82, 055804 (2010), 1011.2518

[36] A. Caciolli, C. Mazzocchi, V. Capogrosso, D. Bemmerer, C. Broggini, P. Corvisiero, H. Costantini, Z. Elekes, A. Formicola, Z. Fülöp et al., Astron. Astrophys. 533, A66 (2011), 1107.4514

[37] C. Fox et al., Phys. Rev. C 71, 055801 (2005)

[38] J.R. Newton, C. Iliadis, A.E. Champagne, J.M. Cesaratto, S. Daigle, R. Longland, Phys. Rev. C 81, 045801 (2010)

[39] D.A. Scott, A. Caciolli, A. Di Leva, A. Formicola, M. Aliotta, M. Anders, D. Bemmerer, C. Broggini, M. Campeggio, P. Corvisiero et al., Phys. Rev. Lett. 109, 202501 (2012), 1210.6529

[40] A. Di Leva, D.A. Scott, A. Caciolli, A. Formicola, F. Strieder, M. Aliotta, M. Anders, D. Bemmerer, C. Broggini, P. Corvisiero et al., Phys. Rev. C 89, 015803 (2014)

[41] A. Caciolli, D.A. Scott, A. Di Leva, A. Formicola, M. Aliotta, M. Anders, A. Bellini, D. Bemmerer, C. Broggini, M. Campeggio et al., Eur. Phys. J. A 48, 144 (2012), 1210.0327

[42] C.G. Bruno, D.A. Scott, A. Formicola, M. Aliotta, T. Davinson, M. Anders, A. Best, D. Bemmerer, C. Broggini, A. Caciolli et al., Eur. Phys. J. A 51, $94(2015)$

[43] C.G. Bruno, D.A. Scott, M. Aliotta, A. Formicola, A. Best, A. Boeltzig, D. Bemmerer, C. Broggini, A. Caciolli, F. Cavanna et al., Phys. Rev. Lett. 117, 142502 (2016), 1610.00483

[44] J. Blackmon et al., Phys. Rev. Lett. 74, 2642 (1995)

[45] M.L. Sergi, C. Spitaleri, M. La Cognata, A. Coc, A. Mukhamedzhanov, S.V. Burjan, S. Cherubini, V. Crucillá, M. Gulino, F. Hammache et al., Phys. Rev. C 82, 032801 (2010)

[46] Straniero, O., Bruno, C. G., Aliotta, M., Best, A., Boeltzig, A., Bemmerer, D., Broggini, C., Caciolli, A., Cavanna, F., Ciani, G. F. et al., A\&A 598, A128 (2017)

[47] M. Lugaro, A.I. Karakas, C.G. Bruno, M. Aliotta, L.R. Nittler, D. Bemmerer, A. Best, A. Boeltzig, C. Broggini, A. Caciolli et al., Nature Astronomy 1, 0027 (2017) 
[48] C. Iliadis, R. Longland, A.E. Champagne, A. Coc, R. Fitzgerald, Nucl. Phys. A 841, 31 (2010), 1004.4517

[49] F. Cavanna, R. Depalo, M. Aliotta, M. Anders, D. Bemmerer, A. Best, A. Boeltzig, C. Broggini, C.G. Bruno, A. Caciolli et al., Phys. Rev. Lett. 115, 252501 (2015), 1511.05329

[50] R. Depalo, F. Cavanna, M. Aliotta, M. Anders, D. Bemmerer, A. Best, A. Boeltzig, C. Broggini, C.G. Bruno, A. Caciolli et al. (LUNA Collaboration), Phys. Rev. C 94, 055804 (2016)

[51] A. Slemer, P. Marigo, D. Piatti, M. Aliotta, D. Bemmerer, A. Best, A. Boeltzig, A. Bressan, C. Broggini, C.G. Bruno et al., Monthly Notices of the Royal Astronomical Society 465, 4817 (2017)

[52] C. Iliadis, A. Champagne, A. Chieffi, M. Limongi, The Astrophysical Journal Supplement Series 193, 16 (2011)

[53] B. Limata et al., Phys. Rev. C 82, 015801 (2010)

[54] F. Strieder, B. Limata, A. Formicola, G. Imbriani, M. Junker, D. Bemmerer, A. Best, C. Broggini, A. Caciolli, P. Corvisiero et al., Phys. Lett. B 707, 60 (2012)

[55] O. Straniero, G. Imbriani, F. Strieder, D. Bemmerer, C. Broggini, A. Caciolli, P. Corvisiero, H. Costantini, S. Cristallo, A. DiLeva et al., Astrophys. J. 763, 100 (2013), 1211.6661

[56] M. Spite, F. Spite, Nature 297, 483 (1982)

[57] M. Asplund, D.L. Lambert, P.E. Nissen, F. Primas, V.V. Smith, Astrophys. J. 644, 229 (2006)
[58] M. Anders, D. Trezzi, A. Bellini, M. Aliotta, D. Bemmerer, C. Broggini, A. Caciolli, H. Costantini, P. Corvisiero, T. Davinson et al., Eur. Phys. J. A 49, 28 (2013), 1301.7185

[59] M. Anders, D. Trezzi, R. Menegazzo, M. Aliotta, A. Bellini, D. Bemmerer, C. Broggini, A. Caciolli, P. Corvisiero, H. Costantini et al., Phys. Rev. Lett. 113, 042501 (2014)

[60] D. Trezzi, M. Anders, M. Aliotta, A. Bellini, D. Bemmerer, A. Boeltzig, C. Broggini, C.G. Bruno, A. Caciolli, F. Cavanna et al., Astropart. Phys. 89, 57 (2017)

[61] LUNA MV Scientific Proposal, https://luna.lngs.infn.it/images/LUNA-MV-5yproposal.pdf

[62] M. Heil, R. Detwiler, R.E. Azuma, A. Couture, J. Daly, J. Görres, F. Käppeler, R. Reifarth, P. Tischhauser, C. Ugalde et al., Phys. Rev. C 78, 025803 (2008)

[63] D. Robertson, M. Couder, U. Greife, F. Strieder, M. Wiescher, Underground nuclear astrophysics studies with CASPAR, in European Physical Journal Web of Conferences (2016), Vol. 109 of European Physical Journal Web of Conferences, p. 09002

[64] W. Liu, Z. Li, J. He, X. Tang, G. Lian, Z. An, J. Chang, H. Chen, Q. Chen, X. Chen et al., Progress of Jinping Underground laboratory for Nuclear Astrophysics (JUNA), in European Physical Journal Web of Conferences (2016), Vol. 109 of European Physical Journal Web of Conferences, p. 09001

[65] LUNA $400 \mathrm{kV} 2015$ - $2018 \quad$ Proposal, https://luna.lngs.infn.it/images/proposal2008.pdf 